\title{
Multiple orale Papeln und Plaques bei einem Patienten mit Morbus Crohn*
}

\author{
Multiple Oral Papules and Plaques in a Patient with Crohn's Disease
}

Autoren

Institut
M. Meissner, A. Pinter, M. Wolter, F. Ochsendorf, R. Kaufmann

Klinik für Dermatologie, Venerologie und Allergologie, Johann Wolfgang Goethe-Universität, Frankfurt am Main
Bibliografie

Dol http://dx.doi.org/

$10.1055 / \mathrm{s}-0034-1367611$

Akt Dermatol 2014; 40: 292-295

(c) Georg Thieme Verlag KG

Stuttgart · New York

ISSN 0340-2541

\section{Korrespondenzadresse \\ Dr. Markus Meissner}

Klinik für Dermatologie, Venerologie und Allergologie

Klinikum der Johann Wolfgang

Goethe-Universität

Theodor-Stern-Kai 7

60590 Frankfurt am Main

markus.meissner@kgu.de

\section{Zusammenfassung \\ $\nabla$}

Bei einem 55-jährigen Patienten traten innerhalb eines Jahres multiple, teils pflastersteinartig imponierende, asymptomatische Papeln an Zunge, Lippen- undWangenschleimhaut auf. Der Patient wurde wegen eines M. Crohn mit Azathioprin und Glukokortikoiden behandelt. Histologisch

\section{Falldarstellung \\ $\nabla$}

\section{Anamnese}

Ein 55-jähriger Patient stellte sich aufgrund von seit zirka einem Jahr bestehenden, größenprogredienten, weißen Papeln und Plaques an der Mundschleimhaut und Zunge vor. Die Veränderungen waren symptomlos, belasteten aber kosmetisch. Der Patient war wegen eines M. Crohn seit Jahren in internistischer Behandlung. Er wurde mit Glukokortikoiden (teils Budesonid-Schaum, teils oral Decortin in Dosen zwischen 10 und $50 \mathrm{mg} / \mathrm{d}$ ) sowie Azathioprin (bis zu $200 \mathrm{mg} / \mathrm{d}$ ) behandelt. Virusserologien auf HIV und Hepatitis B und C waren negativ.

\section{Klinischer Befund}

An der Ober- und Unterlippe, an der Wangenschleimhaut beidseits, sowie ausgeprägt an der Zunge mit Betonung der Zungenränder fanden sich disseminiert verteilte, multiple, scharf begrenzte, bis zu mehrere Millimeter messende, weißliche bis schleimhautfarbene Papeln, die teils zu größeren Plaques konfluierten ( $\bullet$ Abb. $1 \mathbf{a}, \mathbf{b})$. Dies hatte einen pflastersteinartigen Aspekt.

\section{Histologie}

Histologisch zeigte sich eine polypoide, akanthotisch verbreiterte Plattenepithelhyperplasie mit einer Parakeratose, teilweise mit herdförmigen erosiven Oberflächendefekten. In allen durchge- zeigte sich eine akanthotisch verbreiterte Plattenepithelhyperplasie mit Virus-assoziierten Epithelveränderungen. Unter der Diagnose einer oralen fokalen epithelialen Hyperplasie (Morbus Heck) wurden die Papeln, nach erfolgloser Anwendung von Imiquimod-Creme, mittels $\mathrm{CO}_{2}$ Laser erfolgreich vaporisiert.

führten Biopsien fanden sich Epithelveränderungen mit großen Zellkernen und teilweise paranukleärer Halobildung bis in die obere Epithelschicht ( Abb.2a,b).

Diagnose: Orale fokale epitheliale Hyperplasie (Morbus Heck)

\section{Verlauf und Therapie \\ $\nabla$}

Zuerst wurde bei dem Patienten ein Therapieversuch mit topischer Anwendung von Imiquimod $5 \%$-Creme $3 \times /$ Woche über einen Zeitraum von 6 Wochen durchgeführt. Hierunter besserte sich der Befund nicht. Deshalb wurden die Herde mit einem $\mathrm{CO}_{2}$-Laser in Intubationsnarkose abgetragen ( Abb.3a). Postoperativ erhielt der Patient Chlorhexidin-Mundspüllösungen und passierte Kost. Nach dem Eingriff heilten die Herde innerhalb von 2 Wochen problemlos ab $(\checkmark$ Abb. $\mathbf{3 b}$ ). In den folgenden 3 Jahren traten gelegentlich vereinzelt neue Papeln auf, die dann erneut unproblematisch ambulant in Lokalanästhesie mit dem $\mathrm{CO}_{2}$-Laser vaporisiert werden konnten. Ein derart ausgeprägter Befall trat bei dem Patienten nicht mehr auf und er war mit dem ästhetischen Ergebnis zufrieden.

\footnotetext{
* Erstpublikation in Akt Dermatol 2012; 38: 33-36
} 

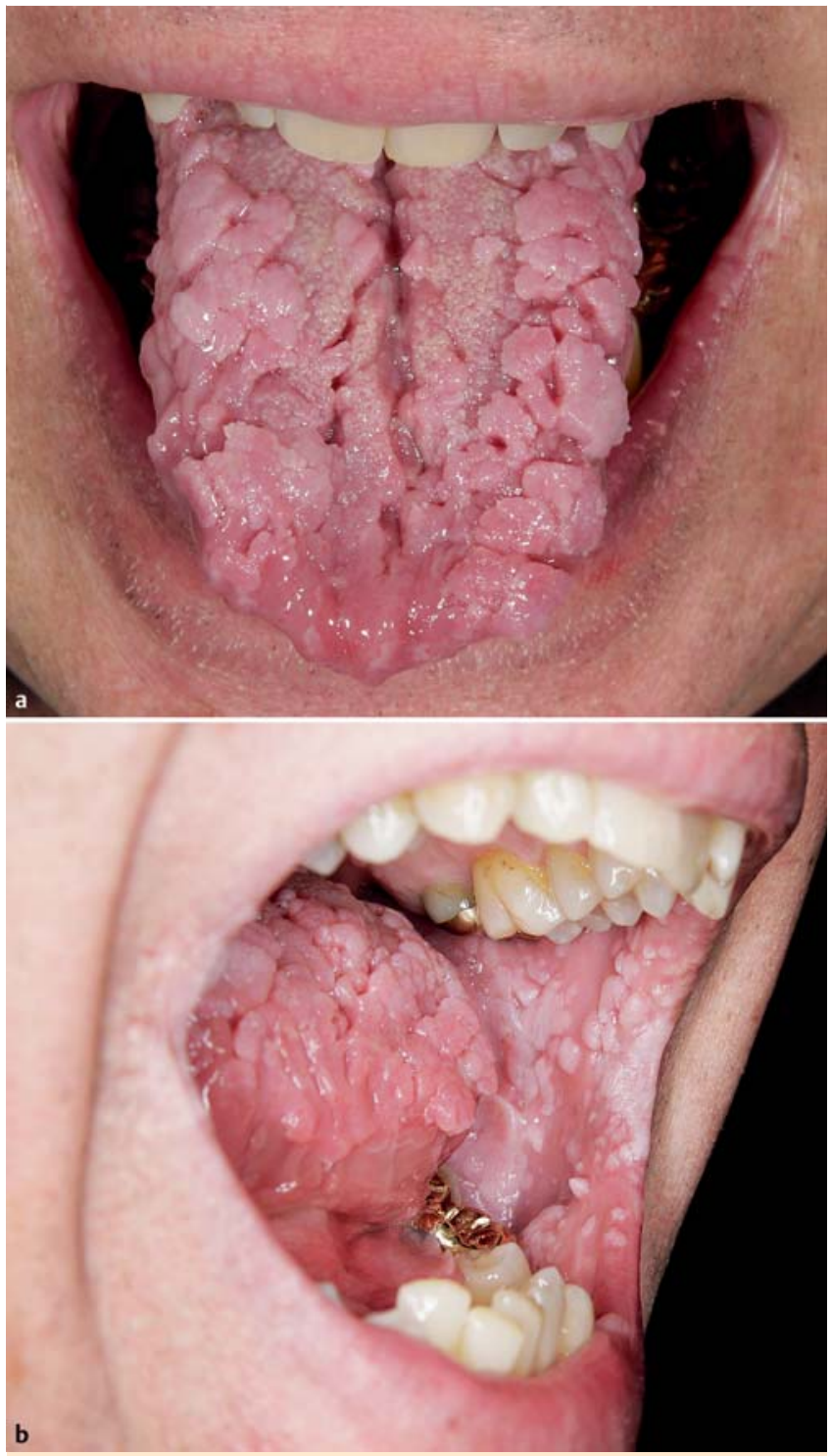

Abb.1 a Multiple, teils konfluierende, weißlich bis schleimhautfarbene Papeln im Bereich der Zunge; b gleiche Hautveränderungen im Bereich der Lippen- und Wangenschleimhaut.

\section{Diskussion}

\section{$\nabla$}

Die orale fokale epitheliale Hyperplasie (FEH) ist eine sehr seltene, gutartige Erkrankung der oralen Schleimhäute, die mit humane Papillomaviren assoziiert ist. Sie wurde erstmals im Jahre 1965 erwähnt und nach Dr. John Heck auch als Morbus Heck benannt [1]. Die Erkrankung tritt vorwiegend bei Kindern und jungen Erwachsenen mit einem Altersgipfel zwischen 9-11 Jahren auf. Sie verläuft chronisch aber meistens innerhalb von Monaten bis einigen Jahren selbstlimitierend. Frauen scheinen etwas häufiger als Männer betroffen zu sein [8]. Besonders häufig ist die fokale epitheliale Hyperplasie bei den Ureinwohnern von Nordund Südamerika, den Eskimos sowie im Süden von Afrika. In Europa oder Asien ist sie dagegen selten. Die genaue Häufigkeit der FEH ist nicht bekannt und scheint je nach ethnischer Zugehörigkeit zwischen 0,002 und 33\% zu liegen [3,6]. Die Klinik zeichnet sich durch multiple, zwischen $2-10 \mathrm{~mm}$ große, teils zu Plaques konfluierende Papeln aus, die teilweise pflastersteinartig imponieren. Die Färbung liegt zwischen weißlich und schleim-

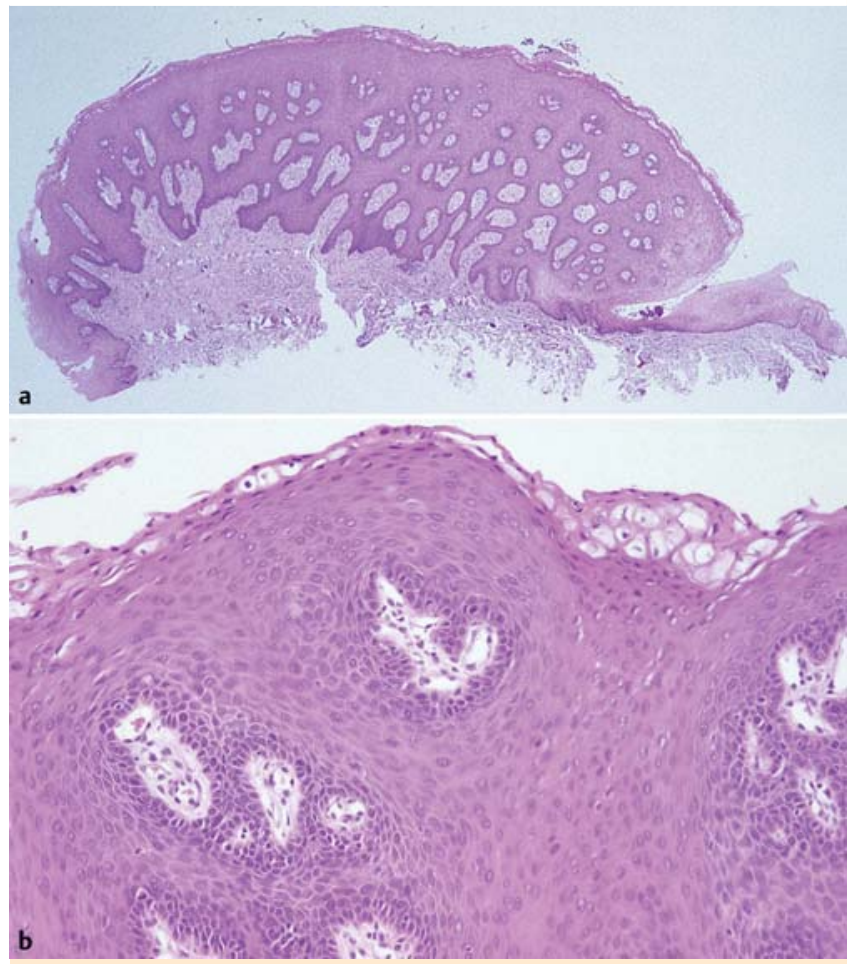

Abb.2 a Übersichtsbild einer probeexzidierten Papel. HE-Färbung ( $20 \times$ Vergrößerung); b Ausschnittbild der akanthotisch verbreiterten Plattenepithelhyperplasie mit Virus-assoziierten Epithelveränderungen mit großen Zellkernen sowie teilweise paranukleärer Halobildung, HE-Färbung (100× Vergrößerung).

hautfarben. Prädilektionsstellen sind insbesondere die Schleimhaut der Lippen, der Wange und der Zunge. Gelegentlich sind auch die Gingiva und die Tonsillenregion betroffen, ganz selten die Genitalschleimhaut. Differenzialdiagnostisch sind beispielsweise an Verrucae vulgares, Condylomata acuminata, eine orale Papillomatose, ein verruköses Karzinom, das Cowden-Syndrom oder eine reaktive fibröse Hyperplasie zu denken [2,7]. Insbesondere die klinische Unterscheidung zwischen der fokal epithelialen Hyperplasie und den Condylomata acuminata kann schwierig sein. Häufig sind die Schleimhautveränderungen bei der FEH zahlreicher und flacher als bei den Condylomata acuminata. Weiterhin ist die FEH meistens auf die Lippen, die Wangen und die Zunge beschränkt. In Zweifelsfällen, insbesondere bei Kindern, bei denen bei Condylomata acuminata auch an einen Missbrauch gedacht werden muss, hilft meistens die komplette Inspektion des gesamten Integuments auf weitere mögliche Zeichen wie Verletzungen, besonderes im anogenitalen Bereich, oder auch das Vorliegen anderer Geschlechtserkrankungen [2].

Pathogenetische Ursache der fokalen epithelialen Hyperplasie scheint eine Virusinfektion mit den humanen Papillomaviren 13 oder 32 zu sein. Cuberso et al. konnten in ihrem Patientenkollektiv von kolumbianischen Kindern in allen Fällen HPV 13 nachweisen, wohingegen in der Kontrollgruppe nur 29,6\% der Kinder HPV 13-positiv waren [4]. HPV 13 scheint im Gegensatz zu HPV 32 relativ spezifisch für die fokale epitheliale Hyperplasie zu sein und kann somit in Zweifelsfällen zur genaueren Diagnostik hinzugezogen werden. Da besonders bestimmte ethnische Gruppen und meistens mehrere Familienmitglieder betroffen sind, geht man davon aus, dass genetische, aber auch Umweltfaktoren eine entscheidende Bedeutung für die Entstehung der fokalen epithelialen Hyperplasie haben. Garcia-Corona et al. konnten in 

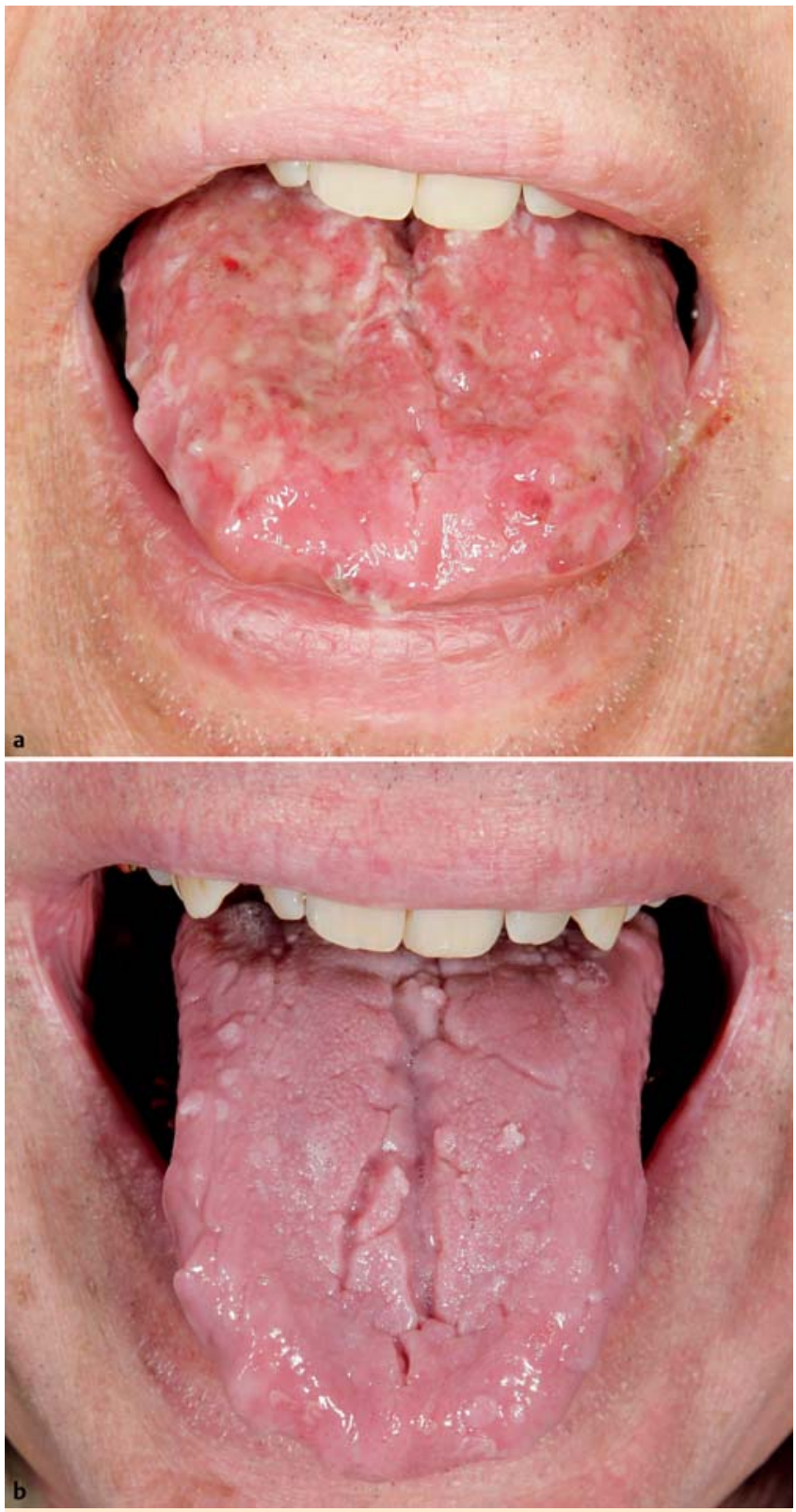

Abb. 3 a Zustand am 3. postoperativen Tag nach CO2-Laserung; b Zustand nach kompletter Abheilung 3 Monate nach Laserung.

einer Studie von 22 Patienten mit FEH nachweisen, dass das Major Histocompatibilitiy Complex (MHC) class II-Gen HLA-DR4 (DRB1*0404) besonders häufig mit der Erkrankung assoziiert ist [6]. Möglicherweise ist dieses Gen, das besonders häufig in der eingeborenen Bevölkerung Amerikas vorkommt, eine wichtige Voraussetzung für die erfolgreiche Infektion mit HPV 13 oder 32. Es wäre somit denkbar, dass das HLA-DR4 (DRB1*0404)-Protein Virusbestandteile des HPV 13 oder 32 schlechter oder gar nicht den zuständigen T-Zellen präsentieren kann.

Das Auftreten der FEH im fortgeschrittenen Alter, wie bei unserem Patienten, kommt sehr selten vor. In den meisten Fällen sind Kinder betroffen. Einen Grund hierfür sieht Ledesma-Montes et al. im möglicherweise noch nicht voll entwickelten Immunsystem der Kinder [8]. Dementsprechend sollte beim Auftreten im Erwachsenenalter an eine immunsuppressive Erkrankung gedacht und diese ausgeschlossen werden. So konnte gezeigt werden, dass in HIV-Patienten ein gehäuftes Auftreten der FEH zu beobachten ist, die sich allerdings paradoxerweise unter einer hochaktiven antiretroviralen Therapie (HAART) verschlechtert [5]. Bei dem eigenen Patienten hat möglicherweise die wegen eines M. Crohn durchgeführte Immunsuppression das Auftreten des M. Heck begünstigt. Die Immunsuppression erklärt möglicherweise auch den nicht erfolgreichen Therapieversuch mit Imiquimod-Creme.

Die Therapieoptionen der FEH beinhalten die klassische Exzision, die Elektrokaustik, die Behandlung mittels $\mathrm{CO}_{2}$ - oder einem 808 nm-Diodenlaser, die Kryotherapie sowie die lokale, systemische oder intraläsionale Gabe von Interferon $\alpha$ oder $\beta$ [7-9]. In letzter Zeit wurde auch gehäuft die erfolgreiche Off-Label-Anwendung von $5 \%$ iger Imiquimod-Creme, die für die Behandlung von HPV-induzierten Condylomata acuminata zugelassen ist, beschrieben [10]. In unserem Fall führte die lokale Anwendung von Imiquimod allerdings zu keiner Besserung, sodass wir die Behandlung mit einem $\mathrm{CO}_{2}$-Laser durchführten. Grundsätzlich kann jedoch in den meisten Fällen, insbesondere bei Kindern, eine abwartende Haltung empfohlen werden, da in einem Großteil der Fälle die Schleimhautveränderungen von selbst narbenlos abheilen.

\section{Fazit für die Praxis}

$\nabla$

- Die orale fokale epitheliale Hyperplasie ist eine seltene, gutartige, meist selbstlimitierende HPV-assoziierte Erkrankung, primär des Kindesalters.

- Beim Auftreten der Erkrankung im Erwachsenenalter sollte an eine immunsuppressive Grunderkrankung gedacht werden.

- Ein Therapieversuch mittels Imiquimod ist eine mögliche Alternative zu den chirurgischen Verfahren.

- Grundsätzlich ist jedoch bei einer hohen Selbstheilungsrate in den ersten 18 Monaten ein zuwartendes Verhalten zu empfehlen.

\section{Abstract}

\section{Multiple Oral Papules and Plaques in a Patient with Crohn's Disease}

$\nabla$

A 55-year old male developed multiple in part cobblestone-like asymptomatic papules at the tongue, the lips, and buccal mucosa. His Crohn's disease had been treated with azathioprine and glucocorticoids. The histology revealed an acanthotic squamous cell hyperplasia with classical virus-associated epithelial changes. After the clinical diagnosis and the ineffective trial of therapy with Imiquimod, a $\mathrm{CO}_{2}$-laser treatment was performed successfully.

\section{Literatur}

1 Archard HO, Heck JW, Stanley HR. Focal epithelial hyperplasia: An unusual oral mucosal lesion found in indian children. Oral Surg Oral Med Oral Pathol 1965; 20: 201-212

2 Bennett LK, Hinshaw M. Heck's disease: diagnosis and susceptibility. Pediatr Dermatol 2009; 26: 87-89

3 Clausen FP. Geographical aspects of oral focal epithelial hyperplasia. Phat-Micobio 1975; 39: 204-213 
4 Cuberos V, Perez J, Lopez CJ et al. Molecular and serological evidence of the epidemiological association of HPV 13 with focal epithelial hyperplasia: a case-control study. J Clin Virol 2006; 37: 21 -26

5 Feller L, Khammissa RA, Wood $\mathrm{NH}$ et al. Focal epithelial hyperplasia (Heck disease) related to highly active antiretroviral therapy in an HIV-seropositive child. A report of a case, and a review of the literature. SADJ 2010; 65: 172 - 175

6 García-Corona C, Vega-Memije E, Mosqueda-Taylor A et al. Association of HLA-DR4 (DRB1*0404) with human papillomavirus infection in patients with focal epithelial hyperplasia. Arch Dermatol 2004; 140: $1227-1231$
7 Hashemipour MA, Shoryabi A, Adhami S et al. Extensive focal epithelial hyperplasia. Arch Iran Med 2010; 13: 48-52

8 Ledesma-Montes C, Vega-Memije E, Garcés-Ortíz M et al. Multifocal epithelial hyperplasia. Report of nine cases. Med Oral Patol Oral Cir Bucal 2005; 10: $394-401$

9 Steinhoff M, Metze D, Stockfleth E et al. Successful topical treatment of focal epithelial hyperplasia (Heck's disease) with interferon-beta. Br J Dermatol 2001; 144: 1067-1069

10 Yasar S, Mansur AT, Serdar ZA et al. Treatment of focal epithelial hyperplasia with topical imiquimod: report of three cases. Pediatr Dermatol 2010; 26: $465-468$ 